\title{
Pour une relecture et un emploi cognitifs des
} schémas narratifs structuralistes

Towards a Cognitivist Conception and a Use of Structuralist Narrative Schemes

\section{Aurora Fragonara}

\section{(2) OpenEdition}

1 Journals

\section{Édition électronique}

URL : http://journals.openedition.org/pratiques/6024

DOI : 10.4000/pratiques.6024

ISSN : 2425-2042

Éditeur

Centre de recherche sur les médiations (CREM)

\section{Référence électronique}

Aurora Fragonara, "Pour une relecture et un emploi cognitifs des schémas narratifs structuralistes », Pratiques [En ligne], 181-182 | 2019, mis en ligne le 30 juin 2019, consulté le 10 décembre 2020. URL : http://journals.openedition.org/pratiques/6024; DOI : https://doi.org/10.4000/pratiques.6024

Ce document a été généré automatiquement le 10 décembre 2020.

(c) Tous droits réservés 


\title{
Pour une relecture et un emploi cognitifs des schémas narratifs structuralistes
}

Towards a Cognitivist Conception and a Use of Structuralist Narrative Schemes

\author{
Aurora Fragonara
}

1 Les travaux structuralistes sur les récits des années 1960 et 1970 ont synthétisé la construction des narrations à l'aide de plusieurs schématisations et modélisations. Nous citerons, à titre d'exemple, le schéma actantiel proposé par A. J. Greimas (1966, nous nous servons de l'édition de 2002), l'analyse des actions et de leurs interactions, proposée par C. Bremond $(1964,1966,1973)$, la macroséquence narrative quinaire, telle qu'elle a été théorisée par P. Larivaille (1974) ou le modèle à intégration progressive des unités de R. Barthes (1977).

2 À l'aune de toutes ces tentatives de modélisation, inspirées par les travaux de V. Propp (1965), et menées sur un large répertoire de contes, les recherches structuralistes de l'époque visaient à dégager des principes et des outils universaux, qui rendraient compte de la structure fondamentale de la narration au-delà de la variété de ses manifestations.

3 Néanmoins, ce dernier point peut faire l'objet de plusieurs critiques et objections. Le fait d'avoir recours à une démarche schématisante, qui ferait abstraction des implications culturelles et anthropologiques, pourrait amener à concevoir et traiter les concepts structuralistes comme un ensemble de postulats normatifs. En d'autres termes, le fait de se servir de ces schémas peut comporter la tentation d'établir une correspondance parfaite et nécessaire entre les modèles et les manifestations factuelles des récits. La pertinence de cette correspondance stricte mérite d'être discutée avant de proposer une autre lecture et un autre emploi des modèles, les deux cognitivement fondés.

4 Comme les questions que nous nous posons ici sont à la fois d'ordre épistémologique et méthodologique, nous allons d'abord discuter le concept de modèle, en exposant les différentes théorisations. Nous présenterons ensuite les réflexions d'U. Eco sur les dimensions ontologique et gnoséologique du concept de structure/modèle dans le 
structuralisme. À partir de cet état de l'art sur la question et ce cadre théorique, nous introduirons et montrerons, à l'aide de l'exemple du Petit Prince, l'intérêt de l'énaction, un concept propre aux sciences cognitives, pour la lecture, l'emploi et la compréhension des récits. Ces réflexions nous permettront de conclure en définissant le statut dont le modèle et le récit factuel sont investis dans une démarche d'analyse énactive.

\section{La notion et la discussion sur les modèles en sciences humaines et sociales}

5 L'approche structuraliste a massivement introduit la notion de modèle en sémiotique (A. J. Greimas, J. Fontanille, U.Eco) et en sciences du langage (l'influence première remonte à R. Jakobson et au cercle de Moscou ; par la suite cette approche est développée par N. Chomsky et S. Harris aux États-Unis ; I. I. Revzin et S. K. Schaumyan en Russie). Quant à la sémiotique, une précision semble importante : parmi d'autres disciplines des sciences humaines et sociales, elle est effectivement celle qui a le plus souvent recours aux modèles. Ayant pour but l'analyse des mécanismes de production de sens lors de toute communication, dans des contextes différents et à travers des supports variés, la sémiotique s'appuie, surtout dans sa phase structuraliste, sur l'activité modélisante suivant des principes logiques. En témoigne les schémas élaborés par A. J. Greimas (carré sémiotique, schéma actantiel, 2002, 1983) et A.J.Greimas et J. Fontanille (structure tensive, 1991). De manière similaire, le recours aux modèles influence la recherche linguistique structuraliste. N. Chomsky et S. Harris, ainsi qu'I. I. Revzin et S. K. Schaumyan, essaient dans leurs études d'opérer un rapprochement entre les modèles mathématiques et linguistiques ${ }^{1}$ (Revzin, 1969, p. 22). Cependant, comme I. I. Revzin le remarque (ibid., p. $28^{2}$ ), la modélisation demeure un sujet de recherche transversal, qui vise plutôt à repérer les conditions et les modalités d'utilisation générales des modèles dans les sciences, au lieu de fournir des exemples concrets de modèles pour chaque discipline particulière.

6 Des chercheurs issus de différents domaines se sont effectivement intéressés à l'activité modélisante et à l'emploi des modèles: l'étude de ces derniers et de leur rapport à la réalité factuelle a fait l'objet de travaux en philosophie des sciences (Heisenberg, 2013; Kuhn, 1983 ; Feyerabend, 1979), en sociologie (l'idéaltype de M. Weber [1992]), et en sciences cognitives (Varela, 1996 ; Varela, Thompson \& Rosch, 1993 ; Lakoff, 1988).

7 Loin d'être déroutante, cette variété des approches est féconde parce qu'elle fournit plusieurs angles d'attaque complémentaires sur la question de la modélisation. Cette complémentarité aide à son tour à mieux cerner la question de la portée épistémologique des modèles dans l'activité de recherche. Chaque chercheur qui s'est penché sur la question a en effet souligné des aspects et des problématiques différents de la relation modèle-vérité factuelle, ce qui nous offre un cadre préliminaire détaillé pour notre étude.

$\mathrm{Vu}$ la multiplicité des approches, nous sommes dans un premier temps obligée d'en choisir une afin de dégager la définition de modèle à partir de laquelle nous développons notre analyse. Comme nous nous focalisons sur les mécanismes d'interprétation des récits et que notre visée est cognitive, nous nous appuyons sur les définitions données à l'intérieur de ce champ de recherche.

9 Le glossaire du recueil Sciences cognitives. Diversité des approches ${ }^{3}$ coordonné par M. B. Gordon et H. Paugam-Moisy (1997) synthétise le concept de modèle de la manière 
suivante : un "schéma prenant en compte les caractéristiques, supposées essentielles, d'un système réel, dans un but explicatif ou prédictif. Un modèle ne cherche pas à reproduire de manière exhaustive le fonctionnement ou les caractéristiques d'un système, mais plutôt à rendre compte des aspects considérés essentiels, avec un minimum d'éléments nécessaires » (ibid., p. 254). Le recours dans sa définition aux mots génériques et hyperonymiques tels que " caractéristiques », « aspects » et « éléments ", témoigne du fait que le modèle est un outil méthodologique applicable à large échelle dans plusieurs domaines, dans différents contextes et suivant des approches variées. La rigueur scientifique des modèles est ainsi confirmée par le degré d'abstraction de la représentation et par l'essentialité des traits qui la composent. Le processus d'abstraction et la recherche des caractéristiques essentielles sont en effet deux modalités de raisonnement à la base de la recherche scientifique (Kuhn, 1983, p. 49-51). Par ailleurs, ces deux caractéristiques sont repérables dans des modélisations présentées et employées dans plusieurs domaines. Par exemple, pour ce qui est de la linguistique, elles sont mentionnées par M.Gross (1968, p. 4-7) comme critères constitutifs des modèles linguistiques de tradition américaine (N.Chomsky, S. Harris) et russe (I. I. Revzin, S. Z. Schaumyan).

10 Une fois les traits distinctifs - abstraction et essentialité - établis, nous pouvons interroger leur rapport avec la réalité factuelle des objets qu'ils cherchent à décrire. Les auteurs que nous avons mentionnés précédemment (W. Heisenberg, M. Weber, T. S. Kuhn) s'accordent sur l'impossibilité et sur la non-nécessité d'une adhésion parfaite entre l'objet de connaissance, tel qu'il se présente dans la réalité, et le modèle de référence ${ }^{4}$. Les conclusions qu'ils en tirent sont variées. Pour W. Heisenberg (2013, p. 99), qui reprend les études de N. Bohr, les modèles dépendent des points de vue qu'on adopte vis-à-vis d'un objet de connaissance, il s'ensuit qu'il est impossible pour un modèle de rendre compte de la totalité des facettes et des angles d'attaque possibles pour l'objet étudié. Ainsi, les modélisations dégagées des points de vue différents sont complémentaires. L'interaction entre elles n'est pas préétablie, mais indéterminée et définissable selon l'angle d'attaque, et, par conséquent, le modèle, que le chercheur choisit au début de son analyse. M. Weber justifie ses propos sur l'idéaltype de la même manière: le modèle idéal (idéaltype), proposé comme piste pour l'analyse des faits sociaux, n'est qu'une hypothèse de travail reformulant les traits communs à un ensemble de phénomènes empiriques appartenant à la même catégorie ${ }^{5}$. Par conséquent, l'idéaltype n'est pas conçu dans le but de représenter fidèlement et entièrement la réalité factuelle d'un phénomène empirique, mais plutôt dans celui de fournir un outil de comparaison pour l'analyse et la vérification des hypothèses préalables. L'activité d'analyse, ainsi que le modèle subjacent, dépendent de l'analyste qui sélectionne et ordonne les données empiriques qui forment les traits essentiels du modèle. Dans cette perspective, T. S. Kuhn (1983, p. 46) remarque que les modèles ont tendance à se cristalliser au sein d'une communauté scientifique donnée: les modèles sont ainsi admis dans la mesure où ils répondent de manière optimale à une question ou à un problème. Cette optimisation n'implique pas forcément une représentation fidèle et totale de l'objet expérientiel, par conséquent le modèle n'est pas chargé d'expliquer tous les faits auxquels il est confronté. Le critère du choix d'une théorie comme la « bonne théorie » repose en grande partie sur les décisions de la communauté scientifique (ibid., p. 30). En soulignant le rôle des chercheurs, T.S. Kuhn établit ainsi un parallèle entre le modèle dominant et celui socialement accepté (ibid., p. 40). Cependant, ces critères du choix optimal et de l'acceptation sociale du modèle ne comportent pas seulement des avantages. Le risque 
majeur observé par T. S. Kuhn est la répétition et la conformation des observations et des expériences au modèle, qui devient par ce fait normatif (ibid., p. 155). Dans ce cadre statique, une révolution scientifique consiste en une rupture du paradigme établi et en son remplacement par un autre (ibid., p. 133) qui parvient à s'imposer après avoir été adopté par un petit groupe innovateur. Le nouveau modèle peut se présenter soit comme radicalement opposé au vieux, soit comme un nouvel accès qui permet de connaitre et étudier un ensemble de données et de problèmes inédits, que la discipline n'avait pas abordés à son stade précédent (ibid., p. 137). Quand (et si) cette nouvelle idée parvient à s'imposer au sein de la communauté scientifique, elle est qualifiée de révolution scientifique, parce qu'elle bouleverse et remplace les fondements de la discipline (ibid., p. 135-138) et instaure une nouvelle vision, et, par conséquent, un nouveau rapport au monde.

P. Feyerabend a également repris cette critique de T.S. Kuhn à l'encontre d'une conception rigide et dogmatique des modèles. En partant du principe que toute idée et théorie sont à priori et potentiellement susceptible d'être passée au crible de la conscience spéculative du chercheur (Kuhn, 1983, p. 21-22), il se peut que les potentiels modèles novateurs existent déjà avant leur affirmation lors d'un moment de crise de la discipline. Les ruptures et remplacements s'inscrivent ainsi dans un flux d'idées dont certaines, abandonnées temporairement, émergent successivement en vertu de leur capacité à fournir des réponses adéquates aux interrogations les plus critiques (Feyerabend, 1979, p. 44). Cette conception de la pluralité des idées et des approches, ouvrant sur une pluralité des questionnements, a été reprise par N. Bouleau dans La modélisation critique (2014). N. Bouleau (ibid., p. 157) souligne la nécessité pour les sciences d'élaborer des modèles "presque vrais» qui admettent l'existence et la validité d'autres modèles, en mesure d'expliquer des aspects non saisis par une seule modélisation.

Cette brève synthèse a montré que les contributions autour du thème des modèles sont nombreuses et variées et que des angles d'attaques différents mènent à des réflexions et à des conclusions partagées. Avant d'approfondir la discussion sur les modèles et sur leur emploi dans notre domaine d'intérêt (la sémiotique), il est donc utile de résumer sous forme de points les concepts issus des auteurs cités précédemment :

1. Le facteur humain (nous dirons plus loin cognitif) est prééminent dans l'activité de modélisation.

2. Les modèles sont indispensables à la recherche et à la progression des connaissances parce qu'ils opèrent une abstraction de l'ensemble des données empiriques.

3. Cependant, leur utilisation comporte un risque majeur: l'assomption essentialiste du modèle et la fossilisation des connaissances qui s'ensuit. Le modèle, d'instrument de connaissance, devient le seul arbitre. Pour être légitimé, un objet doit rentrer dans la « boite » définie par le modèle.

4. Le changement du modèle modifie la vision du monde, c'est-à-dire l'angle d'attaque et l'activité interprétative du chercheur.

13 Comme nous l'avons mentionné dans l'introduction, nous nous intéressons surtout au risque exposé au point 3. Dans la section suivante nous présentons les réflexions d'U. Eco quant à l'approche essentialiste et gnoséologique des modèles/structures dans le courant sémiotico-structuraliste. Cette réflexion nous permettra d'introduire par la suite le concept d'énaction. 


\section{Les modèles en analyse sémiotique et leurs limites : la Structure absente d'Umberto Eco}

14 La réflexion sémiotique est concernée par les questions exposées ci-dessus dans la mesure où elle cherche à dégager des modèles de production du sens, communs à plusieurs substances sémiotiques différentes. Par conséquent, les modèles sémiotiques peuvent être interrogés de la même manière que les modèles étudiés par les philosophes de la science cités ci-dessus. L'interrogation principale s'articule autour de leur représentativité du processus cognitif de création du sens. Dans notre cas spécifique, le champ d'application qui est visé est celui des objets culturels résultant d'un processus de narration. Ces objets présentent une différence par rapport aux cas d'étude choisis par W. Heisenberg, T.S. Kuhn et P. Feyerabend: ils s'inscrivent dans la catégorie des objets sémiotiques (récits, tableaux, publicités, etc.) qui sont produits par des êtres humains et sont analysés par des êtres humains ; alors que dans les sciences dures l'esprit s'attache à l'analyse de phénomènes qui existent indépendamment de son activité créatrice. Cependant, dans les deux cas, la posture de l'analyste est similaire : il emploie le modèle en tant que grille pour l'interprétation et l'analyse des données empiriques et factuelles auxquelles il est confronté (dans notre cas : les récits).

Les questionnements posés par T. S. Kuhn concernant le danger d'un emploi normatif et essentialiste du modèle ainsi que le problème du traitement des écarts par rapport à un modèle demeurent ainsi légitimes.

De manière analogue aux travaux de T. S. Kuhn sur les sciences dures, U. Eco s'est penché sur l'emploi des modèles en sémiotique. Sa réflexion, exposée dans la Struttura assente (1968; La Structure absente dans sa traduction française), se déploie dans la période éminemment structuraliste de la sémiotique.

17 C'est précisément la nature des structures sémiotiques qu'U. Eco questionne, en opposant sa réflexion à la théorisation de C. Lévi-Strauss. Dans la Structure absente, U. Eco pointe ce qui est, selon lui, l'un des dangers majeurs du structuralisme au niveau philosophique et épistémologique: l'identification trop hâtive entre les niveaux gnoséologique et ontologique de la connaissance. Il envisage ce risque dans certains passages des travaux de C. Lévi-Strauss $(1964 ; 1974)$ où les structures employées afin d'expliciter et d'expliquer les contes sont traitées comme consubstantielles à la structure de l'esprit humain ${ }^{6}$.

À l'instar des philosophes des sciences cités dans le paragraphe précédent (M. Weber, W. Heisenberg, T. S. Kuhn et P. Feyerabend), U. Eco repère un clivage entre la conception cognitive (le niveau gnoséologique où se situent les structures de la connaissance proposées par le structuralisme) et la conception empirique des structures (le niveau ontologique, la réalité factuelle d'un objet de connaissance, en termes kantiens la « chose telle qu'elle est»). Contrairement aux affirmations de C. Lévi-Strauss, U. Eco (2008, p. 295-302) soutient que ces structures n'atteignent pas le niveau ontologique, ni dans le cas de l'esprit humain (identifié par C. Lévi-Strauss avec l'inconscient structurel, à valeur ontologique), ni dans le cas de la réalité factuelle des phénomènes. Le fait d'avoir repéré certaines structures générales abstraites permettant d'expliquer et d'expliciter la nature d'un grand nombre d'objets réels revêt un rôle opératoire, et non pas ontologicosubstantiel (ibid., p. 253). D'après U. Eco (ibid., p. 5 de l'introduction), il s'ensuit que les structures ne disent pas comment des objets sont réellement, mais fournissent des outils 
d'analyse pour les appréhender: elles forment ainsi une méthodologie. À ce propos, U. Eco cite l'exemple du modèle d'atome proposé par N. Bohr: il s'avère être une modélisation conçue, élaborée et proposée par un scientifique afin d'expliquer la structure d'un objet de connaissance antécédent au modèle. Le modèle de N. Bohr est ainsi un des modèles possibles, mais il n'est pas ontologiquement nécessaire afin de rendre compte de la structure atomique. Cette réflexion sur le modèle de N. Bohr inscrit les travaux d'U. Eco dans le sillage de T.S. Kuhn et P. Feyerabend sur la pluralité des modèles possibles et sur leur caractère gnoséologique. Elle révèle également le danger du structuralisme ontologique : l'emploi normatif et essentialiste des modèles qui établit une norme et exclut de l'analyse tous les détails et les écarts qui ne sont pas conformes. La vraie et unique structure est ainsi absente, située à l'extérieur de l'esprit du sujet qui, à chaque fois qu'il se penche sur un objet de connaissance, projette sur l'objet une grille modélisante propre à sa manière de penser.

L'objection d'U. Eco et le clivage gnoséologique-ontologique sont importants pour notre analyse car ils légitiment l'existence et la prise en compte des écarts par rapport à la norme. Toutefois, à notre sens, en établissant un clivage net entre ontologie et gnoséologie, la réflexion d'U. Eco sur les structures modélisantes ne s'intéresse pas suffisamment à la dimension cognitive, c'est-à-dire du rapport de connaissance de l'esprit au monde empirique. Ce rapport est d'autant plus important que les récits ne préexistent pas à l'activité de création de l'esprit humain. Si on considère le récit en tant qu'objet de connaissance pour notre analyse et qu'on le pose comme existant, force est de constater que son existence est due premièrement à l'activité d'un esprit et ensuite au processus d'interprétation d'un autre esprit. Ce processus de création implique une organisation, un schéma (et donc un modèle) que l'auteur-créateur met en œuvre au moment de la création, ce même modèle peut être intelligible ou pas pour un destinataire spécifique, selon les cultures. Il s'ensuit que, dans le cas d'une création, les structures ne sont pas seulement gnoséologiques puisqu'elles permettent la réalisation factuelle de l'objet-récit. Elles franchissent ainsi la frontière de la gnoséologie pour s'aventurer dans un territoire ontologique. Cette articulation créatrice gnoséologie-ontologie a été étudiée dans le contexte des sciences cognitives, surtout grâce à la théorie de l'énaction, élaborée par F. Varela (Maturana \& Varela, 1980 ; 1994 ; Varela, 1996; Varela, Thompson \& Rosch, $1993)^{7}$. Le recours à cette théorie de l'énaction nous permet d'élucider le rapport espritréalité, en proposant une définition des fondations de l'esprit humain. Elle est importante non seulement pour la réflexion autour des modèles, mais également pour la validité de l'analyse. En effet, sans un principe fédérateur et unifiant, les résultats risquent d'être trop exposés au côté subjectif propre à toute activité d'interprétation. Une voie « intermédiaire » se profile donc comme nécessaire pour en garantir la rigueur. Dans le paragraphe suivant, nous illustrons le concept d'énaction de manière plus détaillée, en interrogeant davantage les modalités de connaissance propres à l'esprit humain face aux objets qu'il crée ou appréhende, ainsi qu'aux contextes de production et de réception des objets en question.

\section{L'énaction : une voie intermédiaire dans la conception et l'approche des modèles}

Dans tout processus de création et d'interprétation deux éléments sont mobilisés : d'un côté, l'esprit humain et de l'autre l'objet de connaissance, extérieur à la conscience ${ }^{8}$. Le 
rapprochement entre ces deux domaines a été opéré par les sciences cognitives, surtout grâce à la notion d'énaction, telle qu'elle a été développée par F. Varela (1996; Varela, Thompson \& Rosch, 1993). Cette approche met sur un pied d'égalité, et en interaction perpétuelle, les deux entités: le système cognitif et le monde extérieur (qui forme l'ensemble des objets de connaissance). Les objets et l'être humain agissent dans une optique de codétermination mutuelle (Varela, 1996, p. 104 ; Varela, Thompson \& Rosch, 1993, p. 211), où plusieurs facteurs sont mobilisés de la part de l'esprit: le système sensori-moteur ainsi que l'attitude conceptualisante, les opérations d'imagination et l'abstraction de l'esprit humain, (Johnson, 1987, p.14; Lakoff, 1988, p.130-131; Fauconnier \& Turner, 2002, p. 6). Cet « apparatus » de l'être humain se définit et définit la réalité de manière continue grâce à l'interaction avec le monde extérieur: l'action s'oriente par rapport aux données extérieures perçues mais, en vertu de l'organisation du système de perception, elle façonne en même temps le monde extérieur avec lequel elle entre en contact (Varela, Thompson \& Rosch, 1993, p. 234 ; Merleau-Ponty, 1977, p. 11-12).

L'instrument de cette interaction est le corps humain (Varela, Thompson \& Rosch, 1993, p. 123), qui non seulement constitue l'interface de la conscience avec le monde réel, mais enregistre également l'historique de cette relation au monde (Varela, 1996, p. 111). Par conséquent, le concept d'interaction implique également la prise en compte des facteurs environnementaux et socioculturels qui forment le vécu du sujet connaissant (Varela, Thompson \& Rosch, 1993, p. 234). Ces facteurs environnementaux et socioculturels sont déterminés à leur tour de manière interactionnelle, grâce à l'échange entre la réalité socioculturelle et l'« apparatus » cognitif (Varela, 1996, p. 113). Suite à cette interaction, et, potentiellement, à cette redéfinition perpétuelle des relations entre l'être humain et le monde, certains schémas de comportement émergent, se codifient et se réitèrent sans prédétermination définie et nécessaire mais simplement parce qu'ils sont viables (ibid., p. 111) : ils nous permettent de saisir et d'organiser les données provenant du monde extérieur et d'agir dans ce monde par le biais de notre corps et de nos actions (Varela, Thompson \& Rosch, 1993, p. 236).

Rapportée aux sciences humaines et sociales, la perspective de l'énaction permet non seulement de combler la fracture entre l'aspect gnoséologique et ontologique, grâce à la notion d'interaction muable et sans prédétermination, mais également d'identifier un terrain commun pour les deux points de vue, celui du producteur et celui du récepteur d'un même produit culturel : les deux possèdent la même configuration cognitive et, s'ils sont plongés dans le même environnement socioculturel, ils ont intériorisé les mêmes schémas réitérés (ibid., p. 243).

Le schéma actantiel étant un modèle censé rendre compte de la structure des récits, il peut être considéré comme le résultat de cette interaction entre notre système cognitif et un contexte socioculturel particulier dans lequel la production, la lecture et compréhension d'un récit ont lieu. Il est par conséquent un modèle énacté: les composantes et la combinatoire de ces éléments que les lecteurs saisissent et schématisent ont été historiquement définies et organisées par des sujets producteurs (les auteurs) partageant avec les sujets récepteurs (les lecteurs) la même prédisposition cognitive et incarnée ainsi que le même contexte socioculturel (Bremond, 1973, p. 309). Le résultat est un schéma viable et réitéré, qui fonde son existence d'un côté sur une connaissance incarnée ${ }^{10}$ et de l'autre sur des habitudes culturelles et sociales. La preuve est la variabilité des schémas en fonction de l'aire culturelle de référence : nous citons à titre d'exemple, l'expérience menée par W. Kintsch et T. A. Van Dijk (1975) dans deux 
universités, l'une américaine et l'autre néerlandaise, qui montre comment les étudiants occidentaux appliquent les schémas d'analyse issus de leur tradition culturelle à un conte amérindien, structuré selon une combinatoire énactée différente. Cette variété des schémas montre la non-exclusivité ontologique du modèle face à la structuration et à la connaissance du monde, qui jaillit de "l'interface entre l'esprit, la société et la culture» (Varela, Thompson \& Rosch, p. 243).

Puisqu'ils sont fondés sur une interaction et une réitération au sein d'une communauté socioculturelle, les fondements de vérité de ces schémas modélisants ne résident pas dans leur caractère universel et immuable comme le soutenait C. Lévi-Strauss, mais dans leur partage : les structures subjacentes à un récit possèdent une valeur de vérité non pas parce qu'elles sont fixes et universelles, mais parce qu'elles sont partagées entre les auteurs et les lecteurs appartenant à une culture donnée. Le schéma actantiel est ainsi l'une des différentes activations, vraies et possibles, mise en place par notre esprit lorsque nos facultés sont sollicitées par l'objet extérieur « conte».

La substitution du concept d'universalité au profit de celui de partage comporte également une conséquence majeure en matière du traitement des écarts par rapport à la norme. Cette conception énactée du schéma permet en effet d'appréhender différemment non seulement les grandes variations du schéma, dues à la variation culturelle, mais également les petites variations à l'intérieur d'un même contexte socioculturel ${ }^{11}$. Ces menues modifications acquièrent un statut ontologique en vertu de la non-exclusivité et non-nécessité du schéma modélisant de base. Elles ne nécessitent pas d'être normalisées ou d'être justifiées par rapport à la norme : elles peuvent être reconnues et appréhendées comme des composantes spécifiques (donc non-partagées) de la structure d'un récit particulier. Le modèle non-nécessaire permet ainsi de fonder - sur un plan ontologique l'existence de l'écart par rapport à un modèle connu et partagé.

Dans le paragraphe suivant, nous montrons une application possible de la démarche énactive à partir du récit du Petit Prince auquel nous appliquons le schéma actantiel.

\section{Analyser Le Petit Prince en suivant une démarche énactive}

Les opérations énactives qui sous-tendent l'analyse des récits peuvent être montrées à l'aide d'un récit et d'un schéma à appliquer. Pour cette contribution, nous avons choisi d'étudier Le Petit Prince d'A. de Saint-Exupéry en lui appliquant le schéma actantiel.

L'intérêt d'appliquer cette schématisation réside dans la possibilité de faire émerger la structure profonde du récit, qui forme le substrat de l'histoire (Klinkenberg, 2000). Le schéma actantiel regroupe et systématise les personnages sous l'étiquette des actants. Il permet d'identifier les fonctions essentielles d'un récit ainsi que l'ensemble des relations que tissent les composantes de la narration. Il postule enfin la recherche (la quête) d'un objet de la part d'un sujet.

Quant au choix du conte d'A. de Saint-Exupéry, il est motivé par certaines des spécificités narratologiques, textuelles et énonciatives qu'il présente et qui nous permettent de réfléchir au rapport entre le schéma et ses variations. 

fréquents au processus d'écriture et au conte imprimé qui est le résultat de cette volonté d'écrire. La présence de ces éléments permet de considérer cette partie comme une mininarration sur la narration proprement dite du conte. Il s'en suit que les contenus de l'introduction et de l'épilogue métanarratologiques peuvent être synthétisés et expliqués à l'aide du schéma actantiel, qui fait du récit proprement dit l'un des actants :

\section{Schéma 1. Schéma métadiégétique du Petit Prince}

Destinateur: la perte de mémoire dénoncée par l'auteur, qui le pousse à écrire

Ces deux parties forment non seulement un encadrement métadiégétique du récit, mais se structurent à leur tour comme un mini-récit. Il y figure en effet l'explication des

Desinater : la perte de mémoire dénoncéeparl'auteur, qui le pousseàectire

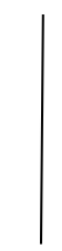

Sujet: le narrateur-aviateur
Adjuvants :
1. Mémoire
2. Langage
3. Dessins

Sujet: le narrateur-aviateur

Objet: le récit, tel qu'il se présente aux destinataires

|

Destinataire: l'aviateur-narrateur qui peut garder le souvenir de son ami et les lecteurs qui pourront connaitre l'histoire, et l'enseignement, du Petit Prince.

Ce premier schéma actantiel représente une variation par rapport au schéma traditionnel, qui traite les éléments du récit, mais n'inclut pas le récit en tant que tel dans le schéma.

Au fur et à mesure qu'on progresse dans l'analyse, le conte montre d'autres variations par rapport au schéma en question. En effet, le récit s'articule autour de deux protagonistes, l'aviateur (qui est également l'auteur/narrateur) et le Petit Prince. Il s'agit des deux personnages qui accomplissent des actions dans un but précis et dont l'état (mental et/ou physique) final est transformé à la fin du récit. De plus, les points de vue adoptés pour la narration coïncident, à tour de rôle, avec la perspective de ces deux personnages. Ces premières considérations nous amènent à un dédoublement du schéma actantiel afin de résumer les quêtes respectives du Petit Prince et de l'aviateur. 
Schéma 2. Schéma actantiel de la quête du Petit Prince

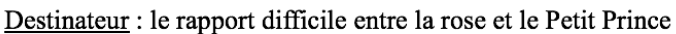

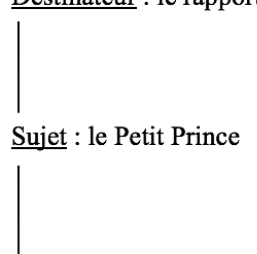

Objet : des réponses sur les rapports interpersonnels. Pour comprendre les personnes, il faut savoir voir au-delà des apparences. (Cette quête, issue des difficultés de compréhension avec la rose, est élargie à une réflexion sur la source de la vérité des choses. Cette source, invisible, jette une lumière nouvelle sur la question des relations interpersonnelles.)

Adjuvants : opposant: l'aviateur

1. habitants des planètes

2. les roses de la roseraie

3. le renard (mentor)

4. l'aiguilleur

5. le marchand de pilules contre la soif

6. le serpent

7. la fleur du désert

Destinataire : le rapport entre le Petit Prince et la rose

Schéma 3. Schéma actantiel de la quête de l'aviateur

Destinateur : l'expérience d'un véritable rapport d'amitié, qui ne soit pas superficiel comme ceux avec les grandes personnes.

Sujet : l'aviateur

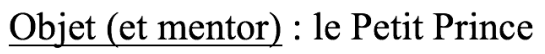

Destinataire : L'aviateur qui découvre la valeur authentique des choses

( l'essentiel est invisible pour les yeux ») et la vérité sur les rapports humains.

Le résultat de cette analyse montre que le Petit Prince est à la fois sujet de sa propre quête et objet/mentor pour la quête de l'aviateur. Ainsi, le fait, pour le Petit Prince, de posséder sa propre quête le place en opposition à l'aviateur. La quête du pilote/narrateur peut être entièrement réalisée seulement si le Petit Prince, devenu son ami, reste sur Terre. Néanmoins, l'objectif à atteindre pour ce dernier exige son départ afin de pouvoir 
rentrer sur son astéroïde. Les deux trajectoires narratives sont donc conflictuelles et peuvent être schématisées de la manière suivante :

Schéma 4. Intersection des trajectoires narratives

\section{Suj.1 = l'aviateur $\quad$ Obj.1 = le Petit Prince

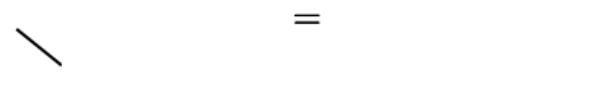

versus
Suj. $2=$ le Petit Prince Obj. $2=$ la rose dans l'ensemble des structures énactées. Comme nous l'avons souligné, elles ont également une fonction de synthèse : dans le cas des modèles proposés pour analyser les contes, le fait de gommer les aspects particuliers et individuels de chaque objet d'analyse permet de reconnaitre les «caractéristiques supposées essentielles " (Mirta \& PaugamMoisy, 1997, p. 254) et de regrouper, de manière synthétique et sous la même étiquette de " récit », des narrations très différentes (littérature, cinéma, théâtre, bande dessinée...). Ce récit modélisé se configure ainsi comme le «tronc structurel commun » (Greimas, 1983, p. 158) qui permet de s'orienter dans le domaine de la narration. Cette schématisation est le résultat d'un acte d'abstraction, qui comporte une certaine rigidité 
et simplification par rapport à la variété des objets sémiotiques observés. La restriction opérée est ainsi fonctionnelle à l'emploi et à la place d'un modèle au sein d'une méthodologie d'analyse et d'observation. En d'autres termes, ce processus à visée synthétique est un système cognitivement viable. Le schéma actantiel réduit effectivement la multiplicité des personnages à des catégories-fonctions, les actants, et regroupe également les actions selon trois axes (désir, communication, pouvoir). Cette classification est pertinente dans l'étude du Petit Prince, qui présente un très grand nombre de personnages, afin d'obtenir une vue d'ensemble sur le récit.

Toutefois, dans le cadre de l'analyse d'un seul récit, une schématisation abstraite n'est pas suffisante. Afin d'étudier sa structure, il est nécessaire d'intégrer les spécificités que ce conte présente et qui ne sont pas mises en valeur dans les schémas modélisants auxquels nous avons recours dans un premier moment. Comme les travaux d'U. Eco l'ont montré, il n'existe aucune contrainte de nécessité ontologique selon laquelle le récit doit obligatoirement reproduire le schéma, au contraire il peut présenter des variations qui contribuent à la création d'un schéma spécifique, propre au récit étudié.

Privés de leur valeur normative, mais inscrits dans une logique de partage au sein d'une communauté, les schémas modélisants se configurent ainsi comme des pôles de comparaison face à un récit, dont la structure subjacente particulière reste à déterminer. Afin de pouvoir dégager ce schéma spécifique, qui rend compte des caractéristiques propres à un récit donné, la comparaison s'avère nécessaire dans le but de relever les similitudes et les différences par rapport au modèle.

Cette démarche comparative se fonde sur une dynamique relationnelle. Les schémas modélisants forment le "pôle 1 » de la connaissance, présentant un système organisé et bien défini d'éléments, alors que le conte visé devient le "pôle 2 ", celui à déterminer. Le passage d'un pôle à l'autre n'implique pas de relation d'identité réflexive et rétroactive, selon laquelle

(le schéma modélisant $\mathrm{du}$ ) Pôle 2 = Pôle 1

$\mathrm{Au}$ contraire, la relation établie est celle de l'émergence au sens cognitiviste du terme. $\mathrm{La}$ comparaison entre le pôle 1 et le pôle 2 vise à faire émerger, et donc à définir de manière libre, la structure du pôle 2, non encore déterminée. Le processus d'émergence d'un schéma par énaction se répète de manière récursive: l'activité cognitive humaine, confrontée à un ensemble d'évènements, fait émerger par énaction des schémas narratifs ; ces schémas, une fois stabilisés et devenus un premier pôle de comparaison, servent, à leur tour, à faire émerger la structure propre à un récit spécifique.

La relation schéma-récit spécifique pourrait ainsi être représentée de la manière suivante :

Pôle $1 \square$ [? (structure)] du pôle 2

45 La valeur du schéma-pôle 1 sera par conséquent heuristique (Odin, 2000, p. 56). Nous considérons ainsi le schéma modélisant en tant que moyen de questionnement de l'objet récit auquel nous sommes confrontés. Cette approche heuristique comporte une analyse qui procède progressivement, à partir d'un constat initial et par tentatives de comparaison avec le pôle 1 , vers un nouveau modèle qui intègre les similitudes et les comparaisons relevées au cours de l'analyse (ibid.).

La rigidité du modèle abstrait (pôle 1) devient ainsi garante d'une connaissance certaine d'un ensemble général de caractéristiques, alors que les caractéristiques propres à l'objet observé (pôle 2) restent encore à élucider. À ce stade l'objet-pôle 2 ne possède aucun 
degré de certitude : il est encore une grandeur sémiotique (Greimas \& Courtés, 1993, p. 339), c'est-à-dire une entité antérieure à l'analyse. Par conséquent, ses caractéristiques sont inconnues et vont ressortir de la comparaison avec le modèle déjà connu. La dynamique de la comparaison jaillit ainsi d'un déséquilibre gnoséologique entre le modèle, abstrait, simplifié et entièrement connu, et l'objet observé dont à ce stade nous avons juste posé un constat d'existence (le récit Le Petit Prince existe, il a été publié et nous avons pu le lire). Dans cette perspective heuristique, le pôle 1 ne se pose pas en tant qu'hypothèse à confirmer, tel que T. S. Kuhn l'avait noté pour la science normale, mais comme point de départ pour appréhender l'analyse du récit. Il s'ensuit que le pôle 2 se développe ensuite de manière autonome et donne des résultats qui ne sont pas forcément attendus et donc conformes au pôle 1 . Toutefois, cette non-conformité demeure l'un des résultats prévus par l'énaction heuristique. Pour cette raison, en cas de non-coïncidence, l'activité de comparaison n'invalide, ni délégitime le modèle-pôle 1 du départ, ni le nouveau-schéma pôle 2. Cette perspective énactive se sert donc des schémas en leur ôtant toute portée ontologique, mais en préservant leur validité gnoséologique, ce qui permet de mener à bien l'analyse.

47 La perspective énactive, explicitée par la dynamique des deux pôles, permet ainsi de montrer la validité du schéma ainsi que des variations qui peuvent être repérées lors de l'analyse des récits.

\section{BIBLIOGRAPHIE}

BARTHES, R. (1977) [1966]. « Introduction à l'analyse structurale des récits ». In : Poétique du récit. Paris : Éditions du Seuil, p. 7-57.

BOULEAU, N. (2014). La Modélisation critique. Versailles : Éditions Quæ.

BREMOND, C. (1964). « Le message narratif ». Communications 4, p. 4-32. En ligne : https:// www.persee.fr/doc/comm_0588-8018_1964_num_4_1_1025.

BREMOND, C. (1966). « La logique des possibles narratifs ». Communications 8, p. 60-76. En ligne : https://www.persee.fr/doc/comm_0588-8018_1966_num_8_1_1115.

BREMOND, C. (1973). Logique du récit. Paris : Éditions du Seuil.

ECO, U. (2008) [1968]. La struttura assente. Introduzione alla ricerca semiologica. Milano :

Bompiani.

FAuConNier, G. \& TURner, M. (2002). The Way We Think. Conceptual Blending and the Mind's Hidden Complexities. New York : Basic books.

FEYERABEND, P. (1979) [1975]. Contre la méthode. Esquisse d'une théorie anarchiste de la connaissance. Trad. de l'anglais par B. Jurdant et A. Schlumberger. Paris : Éditions du Seuil.

FONTANILLE, J. (2003) [1998]. Sémiotique du discours. Limoges : Presses universitaires de Limoges.

GORDON, M. B. \& PAUGAM-MOISY, H. (dirs) (1997). Sciences cognitives. Diversité des approches. Paris : Hermès. 
GREIMAS, A. J. (1983) [1970]. Du sens I-II. Paris : Éditions du Seuil.

GREIMAS, A. J. (2002) [1966]. Sémantique structurale. Recherche de méthode. Paris : Presses universitaires de France.

GREIMAS, A. J. \& COURTÉS, J. (1993) [1979]. Sémiotique. Dictionnaire raisonné de la théorie du langage. Paris : Hachette.

GREIMAS, A. J. \& FONTANILLE, J. (1991). Sémiotique des passions. Des états de choses aux états d'âme.

Paris : Éditions du Seuil.

GROSS, M. (1968). «L'emploi des modèles en linguistique ». Langages 9, p. 3-8. En ligne : https:// www.persee.fr/doc/lgge_0458-726x_1968_num_3_9_2358.

HEISENBERG, W. (2013) [1971]. Fisica e oltre. Trad. par M. e Dida Paggi. Torino : Bollati Boringhieri.

JOHnson, M. (1987). The Body in the Mind. Chicago : University of Chicago Press.

KINTSCH, W. \& VAN DIJK, T. A. (1975). « Comment on se rappelle et on résume des histoires ».

Langages 40, p. 98-116. En ligne : https://www.persee.fr/doc/

lgge_0458-726x_1975_num_9_40_2300.

KLINKENBeRG, J.-M. (2000) [1993]. Précis de sémiotique générale. Paris : Éditions du Seuil.

KUHN, T. S. (1983) [1970]. La Structure des révolutions scientifiques. Trad. de l'anglais (États-Unis) par L. Meyer. Paris : Flammarion.

LAKOFF, G. (1988). « Cognitive Semiotics ». In : Eco, U., Santambrogio, M. \& Violi, P. (dirs), Meaning and Mental Representations. Indianapolis : Indiana University Press, p. 119-154.

LARIVAILlE, P. (1974). «L'analyse (morpho)logique du récit ». Poétique 19, p. 368-388.

LEVI-STRAuSS, C. (1964). Le Cru et le cuit. Paris : Éditions du Seuil.

LEVI-STRAUSS, C. (1974) [1958]. Anthropologie structurale. Paris : Plon.

maturana, H. \& VARELA, F. (1980). Autopoiesis and cognition. The realization of the Living. Boston : Kluwer.

MATURANA, H. \& VARELA, F. (1994). L'Arbre de la connaissance. Rrad. de l'anglais (États-Unis) par F.C. Jullien avec la collab. de H. Trocmé-Fabre. Addison-Wesley : France.

MERLEAU-PONTY, M. (1976) [1945]. Phénoménologie de la perception. Paris : Gallimard.

MERLEAU-PONTY, M. (1977) [1942]. La Structure du comportement. Paris : Presses universitaires de France.

ODIN, R. (2000). «La question du public. Approche sémio-pragmatique ». Réseaux 99, p. 49-72. En ligne : https://www.persee.fr/doc/reso_0751-7971_2000_num_18_99_2195.

PRopP, V. (1970) [1965]. Morphologie du conte. Trad. du russe par M. Derrida, T. Todorov et C. Kahn. Paris : Éditions du Seuil.

REVZIN, I. I. (1969). « Les principes de la théorie des modèles en linguistique ». Langages 15, p. 21-31. En ligne : https://www.persee.fr/doc/lgge_0458-726x_1969_num_4_15_2515.

RICCEUR, P. (1984). Temps et récit, tome I. Paris : Éditions du Seuil.

TURNER, M. (1995). The Literary Mind. The Origins of Thought and Language. New York/Oxford : Oxford University Press. 
VARELA, F. (1996) [1988]. Invitation aux sciences cognitives. Trad. de l'anglais par P. Lavoie. Paris : Éditions du Seuil.

VARELA, F., THOMPSON, E. \& ROSCH, E. (1993) [1991]. L'Inscription corporelle de l'esprit. Sciences cognitives et expérience humaine. Trad. de l'anglais par V. Havelange. Paris : Éditions du Seuil. WEBER, M. (1992) [1904-1917]. Essais sur la théorie de la science. Trad. de l'allemand par J. Freund. Paris : Pocket.

\section{NOTES}

1. Le numéro 15 de la revue Langages (1969), qui contient l'article d'I. I. Revzin cité ici, est entièrement consacré à la réflexion et l'élaboration des modèles linguistiques en dehors de leur contexte de production et énonciation, ainsi qu'à l'articulation entre modèles linguistiques abstraits et modèles mathématiques.

2. "Nous allons nous efforcer de décrire d'une manière plus ou moins exhaustive ce que nous entendons par "modèle" (définir le terme "modèle" est impossible parce que le concept ne se rapporte pas à la théorie linguistique des modèles mais à une métathéorie, laquelle, qui plus est, s'occupe non pas des modèles concrets mais des principes généraux d'application des modèles dans toutes les sciences) ».

3. Les contributions présentées sont issues de différents domaines (neurosciences, linguistique, mathématiques) susceptibles de former la galaxie des sciences cognitives. Dans cette pluralité d'approches de la cognition, le choix d'insérer le mot «modèle » dans le glossaire témoigne du fait qu'il est conçu comme un concept commun et à la base de toutes les contributions qui sont réunies dans le volume : il est un des présupposés de la recherche.

4. Le modèle est appelé différemment selon les auteurs: idéaltype chez M. Weber, paradigme chez T. S. Kuhn.

5. M. Weber s'occupe surtout d'organisations socioéconomiques, il dégage ainsi des modèles de bureaucratie, capitalisme, etc.

6. Cf. ce passage du Cru et le cuit $(1964$, p. 18) : "Plus décisive sera donc l'expérience que nous entreprenons maintenant sur la mythologie. Celle-ci n'a pas de fonction pratique évidente ; à l'inverse des phénomènes précédemment examinés, elle n'est pas en prise directe sur une réalité différente, dotée d'une objectivité plus haute que la sienne, et dont elle transmettrait les ordres à un esprit qui semble parfaitement libre de s'abandonner à sa spontanéité créatrice. Par conséquent, s'il était possible de démontrer que, dans ce cas aussi, l'apparence arbitraire, le jaillissement prétendu libre, l'invention qu'on pourrait croire débridée, supposent des lois opérant à un niveau plus profond, la conclusion deviendrait inéluctable que l'esprit, livré au tête-à-tête avec lui-même et échappant à l'obligation de composer avec les objets, se trouve en quelque sorte réduit à s'imiter luimême comme objet; et que, les lois de ses opérations n'étant pas alors fondamentalement différentes de celles qu'il manifeste dans l'autre fonction, il avère ainsi sa nature de chose parmi les choses. Sans pousser aussi loin le raisonnement, il nous suffira d'avoir acquis la conviction que si l'esprit humain apparaît déterminé jusque dans les mythes, alors a fortiori il doit l'être partout ». Et encore dans Anthropologie structurale (1974, p. 34) : « En ethnologie, comme en linguistique, par conséquent, ce n'est pas la comparaison qui fonde la généralisation, mais le contraire $\mathrm{Si}$, comme nous le croyons, l'activité inconsciente de 
l'esprit consiste à imposer des formes à un contenu, et si ces formes sont fondamentalement les mêmes pour tous les esprits, anciens et modernes, primitifs et civilisés - comme l'étude de la fonction symbolique, telle qu'elle est exprimée dans le langage, le montre de façon éclatante -, il faut et il suffit d'atteindre la structure inconsciente, sous-jacente à chaque institution ou à chaque coutume, pour obtenir un principe d'interprétation valide pour d'autres institutions et d'autres coutumes, à condition, naturellement, de pousser assez loin l'analyse ».

7. En philosophie, dans le cadre de l'approche phénoménologique, des réflexions semblables sont menées par M. Merleau-Ponty $(1976,1977)$.

8. Cet objet extérieur est appelé et catégorisé différemment dans d'autres travaux sémiotiques : J. Fontanille (2003, p. 71-72), parle de la propioception de l'objet, alors que J.-M. Klinkenberg (2000, p. 100-101) traite des fondations extérieures du sens.

9. Nous soulignons au passage que l'aspect culturel de l'organisation d'un récit est également reconnu par P. Ricœur à propos de la "mise en intrigue " en tant que configuration. À plusieurs reprises, il définit l'intrigue en tant que « archétype culturel ", « configuration(s) culturellement reconnue(s) ", ou un "schématisme du récit à l'œuvre dans les intrigues-types reçues de la tradition » (Ricœur, 1984, p. 68). Toutefois, aucune description de la forme de cette intrigue-type ne nous est livrée.

10. Pour le concept de connaissance incarnée nous renvoyons aux travaux de M. Johnson (1987), M. Turner (1995) et M. Tuner et G. Fauconnier (2002). Dans leur ouvrage de 2002, The Way We Think, M. Turner et G. Fauconnier regroupent sous l'étiquette de « relations vitales » l'ensemble des opérations logico-expérientielles qui permettent à l'être humain de vivre et comprendre le contexte dans lequel il est situé. Les relations vitales se spécifient en relations d'intention, d'unicité, transformation, recherche de la cause et compréhension/gestion des contraintes spatio-temporelles.

11. Nous allons voir dans le cas du Petit Prince que des variations moindres peuvent se reproduire à une échelle modeste.

\section{RÉSUMÉS}

L'article propose d'interroger le statut et l'emploi des schémas structuralistes, souvent jugés figés et contraignants, dans l'analyse des récits. Après avoir passé en revue les différentes conceptualisations théoriques des schématisations, nous en interrogeons le statut gnoséologique, à l'aide de la critique de l'ontologisme des structures proposée par U. Eco et du concept d'énaction, qui permet de comprendre l'émergence des schèmes mentaux. Nous expliquons ce fonctionnement énactif à l'aide de l'analyse de la structure narrative du Petit Prince, afin de mettre en évidence la portée heuristique et gnoséologique de la démarche schématisante. Cette illustration montre la validité des modélisations structuralistes, tout en évitant les soupçons de prescriptivisme.

This paper aims to enquire about the status and the use of structuralist theories and schematisations in analysing narratives forms, since schemes are often regarded as restrictive and pre-established. After describing the state-of-the art of the concept of schematisation in 
different domains, we discuss its gnoseological status, referring to U. Eco's critique of the ontology of structuralist presuppositions as well as to the concept of enaction, a cognitive operation that enables the emergence of mental schemes. We consequently show an enactive application of the actantial model to the Petit Prince in order to enlighten the heuristic and gnoseological value of the scheme. The validity of structuralist schemes is thus preserved and, in the meantime, prescriptive suspicions are avoided.

\section{INDEX}

Keywords : structuralism, actantial scheme, absent structure, cognition, enaction, Petit Prince (Le)

Mots-clés : structuralisme, schéma actantiel, structure absente, cognition, énaction, Petit Prince (Le)

\section{AUTEUR}

\section{AURORA FRAGONARA}

Università degli studi di Bergamo, IT-24129 Bergame, Italie 